\title{
Pinpointing the vesper bat transposon revolution using the Miniopterus natalensis genome
}

\author{
Roy N. Platt II, Sarah F. Mangum and David A. Ray
}

\begin{abstract}
Background: Around 40 million years ago DNA transposons began accumulating in an ancestor of bats in the family Vespertilionidae. Since that time, Class II transposons have been continuously reinvading and accumulating in vespertilionid genomes at a rate that is unprecedented in mammals. Miniopterus (Miniopteridae), a genus of long-fingered bats that was recently elevated from Vespertilionidae, is the sister taxon to the vespertilionids and is often used as an outgroup when studying transposable elements in vesper bats. Previous wet-lab techniques failed to identify Helitrons, TcMariners, or hAT transposons in Miniopterus. Limitations of those methods and ambiguous results regarding the distribution of piggyBac transposons left some questions as to the distribution of Class II elements in this group. The recent release of the Miniopterus natalensis genome allows for transposable element discovery with a higher degree of precision.

Results: Here we analyze the transposable element content of M. natalensis to pinpoint with greater accuracy the taxonomic distribution of Class II transposable elements in bats. These efforts demonstrate that, compared to the vespertilionids, Class II TEs are highly mutated and comprise only a small portion of the M. natalensis genome. Despite the limited Class II content, M. natalensis possesses a limited number of lineage-specific, low copy number piggyBacs and shares several TcMariner families with vespertilionid bats. Multiple efforts to identify Helitrons, one of the major TE components of vesper bat genomes, using de novo repeat identification and structural based searches failed.

Conclusions: These observations combined with previous results inform our understanding of the events leading to the unique Class II element acquisition that characterizes vespertilionids. While it appears that a small number of TcMariner and piggyBac elements were deposited in the ancestral Miniopterus + vespertilionid genome, these elements are not present in $M$. natalensis genome at high copy number. Instead, this work indicates that the vesper bats alone experienced the expansion of TEs ranging from Helitrons to piggyBacs to hATs.
\end{abstract}

Keywords: Miniopteridae, Vespertilionidae, TcMariner, Helitron, Transposable element

\section{Background}

Transposable elements (TEs) are genetic elements with the ability to mobilize throughout a host genome. Often TE copies are generated as a result of the mobilization process and TEs can end up occupying large portions of mammalian genomes. For example, between 45 and $70 \%$ of the human genome is occupied by TEs $[1,2]$. TEs are classified into two major classes based on their mobilization

* Correspondence: david.4.ray@gmail.com

Department of Biological Sciences, Texas Tech University, Box 43131, Lubbock, TX 79409-3131, USA mechanism. Class I elements, also known as retrotransposons, mobilize as an RNA intermediate that is reverse transcribed back into the genome. These elements are referred to as "copy and paste" elements since they generate identical copies of themselves upon insertion. Retrotransposons are further classified into Long Terminal Repeats (LTRs), Long INterspersed Elements (LINEs), and Short INterspersed Elements (SINEs). Class II elements, also known as DNA transposons, mobilize via a transposase enzyme. During mobilization, the terminal inverted repeat-containing DNA transposons physically excise from the genome and re-integrate at another locus. 
However, in addition to these canonical "cut and paste" DNA transposons, Helitrons and Mavericks mobilize through other mechanisms that do not fully excise the template TE. As a result, these Class II elements are "copy and paste" transposons since they mobilize through a single DNA strand excised from the parent locus.

In general, retrotransposons are much more common in mammalian genomes than DNA transposons. For example, $43 \%$ of the human genome is derived from retrotransposons vs. $3 \%$ from DNA transposons [2]. In addition to being less frequent, transposons are often found in genomes as heavily mutated insertions; indicating long periods of inactivity. The single major exception to this general trend is the presence of recently inserted Class II elements in the genomes of vespertilionid bats $[3,4]$. As much as $6 \%$ of the Myotis lucifugus genome is derived from recently active Helitrons [5], 3.5 \% from cut and paste transposons [6], and half of all recent TE accumulation appears to come from Class II elements [7].

To understand the timing and evolutionary implications of this unique activity, we must first identify the taxonomic distribution and accumulation patterns of the elements involved. Previous work focusing on the initial horizontal transfer or reactivation of Class II elements in vespertilionids indicated that Helitrons are restricted to the vespertilionid lineage [8] and only a limited number of cut and paste transposon families are found beyond Vespertilionidae [6]. These results were based on comparisons of vespertilionids to several non-vesper bats including Miniopterus, a genus of long-fingered bats recently elevated to familial level from Vespertilionidae [9]. For example, using internal PCR primers, Ray et al. [6] tried to amplify piggyBac, hAT, and TcMariner elements in a panel of chiropteran including Artibeus jamaicensis, Balionycteris sp., Corynorhinus rafinesquii, Eptesicus furinalis, Hipposideros cervinus, Kerivoula papillosa, Macroglossus sobrinus, Miniopterus sp., Myotis austroriparius, My. horsfieldii, Natalus stramineus, Nycticeius humeralis, Pteronotus parnellii, Rhinolophus borneoensis, and Thyroptera tricolor. Results indicated that TcMariner elements were only present in vespertilionids (C. rafinesquii, E. furinalis, K. papillosa, Myotis austroriparius, Myotis horsfieldii, and $N$. humeralis). hATs and piggyBacs were only found in Myotis species, with the exception of one piggyBac (piggyBac2_ML) that was amplified in Myotis sps. and Miniopterus but was absent in other all other samples including the non-Myotis vespertilionids [6]. Probe-based hybridization failed to identify Helitrons in Miniopterus or any other non-vesper bats [8].

Modern genome assembly and sequencing techniques provide many advantages for TE discovery over wet-lab based techniques. Mispriming, in the case of PCR, or reduced hybridization efficiency, in the probe-based analyses, could easily allow elements to be missed in any or all of these genomes. In addition, these methods rely on a priori knowledge of TE content in order to build primers/probes for loci of interest. The recent release of the Miniopterus natalensis genome [10] allows these questions to be answered more precisely and with independent and unbiased data. Here, we characterize the repetitive portion of the $M$. natalensis genome with an emphasis on Class II elements in order to understand the acquisition of these Class II TEs in bats.

\section{Methods}

Repeats were identified in the Miniopterus natalensis genome using de novo methods and TEs were fully validated [11] as detailed below. Putative repeats were identified using RepeatModeler [12] and the current $M$. natalensis assembly (Genbank accession GCA_001595765.1). The RepeatModeler repeats were masked with RepeatMasker [13] using all known Chiropteran TEs (-species "Chiroptera") to remove repeats that have already been described in other bat species. Those repeats that were $\geq 80 \%$ similar to known elements across more than $50 \%$ of their length were excluded from downstream analyses. The remaining elements were considered possible Miniopterus-specific elements. To manually validate these repeats, they were used as BLASTn v2.2.27 [14] queries against the $M$. natalensis genome. BLASTn hits were restricted to those with $\mathrm{E}$ values greater than $1 e-10$. For each repeat, the forty loci most similar to the BLASTn query were extracted from the genome along with 500 or more bases of flanking sequence and aligned using MUSCLE v3.8.1551 [15]. Repeats with less than 10 BLASTn hits were culled from further analysis. For the remaining repeats, majority-rule consensus sequences were generated for each alignment using BioEdit v7.2.5 [16]. Elements that contained single copy DNA on both the $5^{\prime}$ and $3^{\prime}$ end were considered to be complete. If an alignment ended within a repetitive portion, the consensus sequence was generated across the entire repetitive portion of the alignment and this new consensus sequence was used as a query in subsequent BLASTn rounds. This process was iterated until all de novo repeats were fully represented.

Beyond RepeatModeler searches, attempts were made to identify low copy number and highly divergent Helitrons using HelitronScanner [17]. HelitronScanner searches the genome for 5' and 3' terminal sequences associated with Helitrons. Terminal sequences are then paired with their closest partner. Those falling within a set distance are considered putative Helitrons. Default parameters were used in HelitronScanner searches except for the scoring threshold, which was raised from a default of 5 to 10 . As a control, a copy of the M. natalensis genome was shuffled using EMBOSS's shuffleseq (v6.6.0 [18]), and run in parallel using the same parameters. A series of BLAT [19], and BLAST searches were used to 
validate putative Helitrons that resulted from HelitronScanner queries.

All novel repeats were classified based on structural hallmarks (ex. poly-A tails, target site duplications, terminal inverted repeats, etc.) and homology to other TEs present in RepBase (accessed 1 April 2016 [20]). For larger elements, intact open reading frames (ORFs) were identified with ORF Finder [21]. Elements were classified using the 80-80-80 rule [22] and designated based on standard naming conventions implemented by RepBase [20]. For example, two SINEs in M. natalensis meet the 80-80-80 thresholds when compared to the canonical VES SINE, but each varies from one another by $5 \%$ at the nucleotide level and contain diagnostic indels. In this case, both SINEs are recognized as members of the separate subfamilies of VES: VES-1_MNa and VES-2_MNa. After classification, the M. natalensis repeats were combined with all known mammal TEs from RepBase and used as a customized library to annotate the $M$. natalensis genome. For comparative purposes genomes from closely related bat species, were analyzed using identical RepeatMasker settings to provide a better estimate of the TE dynamics during the Miniopterus and Vespertilionidae divergence. These taxa include Myotis lucifugus (GCA_000147115.1), Eptesicus fuscus (GCA_000308155.1) and Pteronotus parnellii (GCA_000147115.1) and were chosen based on their phylogenetic relationships. Repeat accumulation profiles for all taxa were generated using the Kimura 2-parameter distance [23] between the RepeatMasker library and homologous loci in the genome. Highly mutable CpG sites [24] were excluded from distance calculations. Elements belonging to the same superfamily were binned based on their genetic distances. Distances were rounded down to the nearest full percentage. For comparison, average genetic distances between genomic TEs and the consensus library TE were calculated for all DNA transposons occupying more than $10 \mathrm{~Kb}$ of any bat genome.

To identify TEs specific to $M$. natalensis, repeats identified by RepeatModeler and successfully validated, were used as BLASTn queries against all other genomes in the NCBI Genomes (chromosomes) database. M. natalensis was excluded (NCBI Taxa ID 9432) from these searches. The most closely related species to $M$. natalensis in the NCBI Genome database are the vespertilionids, Myotis lucifugus, Myotis brandtii, Myotis davidii, and Eptesicus fuscus. Pteronotus parnellii (family Mormoopidae), serves as an outgroup to a monophyletic clade comprising Vespertilionidae + Miniopteridae [25]. Repeats were classified based on the species distribution of the 50 best BLASTn hits. If the best hits for a repeat belonged to a vespertilionid or $P$. parnellii, the $M$. natalensis repeat was assumed to have been active in the common ancestor of these taxa. If, however, the best hits were to species other than a vespertilionid or $P$. parnellii, then the TE has a distribution among species that does not follow the species tree. If no hits were found to other species, it was assumed that these elements are only found in $M$. natalensis and are lineage-specific. BLASTn hits were only considered if they had an $\mathrm{E}$ value greater than $1 e-10$ and were more than $80 \%$ similar across $80 \%$ of the length of the $M$. natalensis query.

\section{Results}

RepeatModeler analysis of the unmasked Miniopterus natalensis genome identified 396 putative repetitive sequences. After removing elements with homology to known chiropteran TEs, simple repeats, and low copy number elements 52 putative TEs remained. Of these, 13 were so heavily mutated in the $M$. natalensis genome that generating a consensus sequence was not feasible. The remaining 39 elements were fully validated and classified. In all: 10 LTRs, 2 SINEs, 2 LINEs, and 25 DNA transposons were identified. All LTR elements were solo LTRs of less 1,100 bp. These LTRs were classified as ERV1 (gammaretroviruses) or ERV3s (spumaviruses) based on the size of their target site duplicates. The two SINEs were variants of the VES family of SINEs common in many bats [26, 27]. The two LINEs belonged to the LINE-1 superfamily and were full length, with intact ORF2s, but contained premature stop codons in ORF1 of the consensus elements. Three non-autonomous piggyBac elements were recovered and verified via their TTAA target site duplications. Finally, 22 elements in the TcMariner superfamily were identified including three potentially autonomous elements. BLASTp results from ORFs in these transposons revealed similar domain organization in each. ORFs ranged in length from 493 to 594 amino acids and two of the three contained a helix-turn-helix, Tc5 transposase, and DDE-like integrase domain while the third lacked the initial helix-turn-helix domain. All TcMariner elements had terminal inverted repeats of 12-26 bps that began with CAG and TA target site duplications.

HelitronScanner was used to identify low copy number Helitrons that would have been culled based on the filtering criteria for the RepeatModeler data. As a negative control, searches for Helitrons were run in parallel on $M$. natalensis, and a shuffled version of the $M$. natalensis genome. HelitronScanner identified 10 elements ranging in size from 2,351 to $14,820 \mathrm{bps}$ in the $M$. natalensis genome and none in the shuffled genome. Several steps were taken to confirm these as true Helitrons. First, these elements were used as BLASTn queries against the $M$. natalensis genome to determine copy number. Other than the original locus, no significant hits were found indicating these putative Helitrons were single copy. Next, we used BLAT to compare the putative Helitrons to 
the Myotis lucifugus genome. In nine of the 10 cases, fulllength elements were found, but none overlapped with known Myotis lucifugus Helitrons, in the tenth case, no homologous sequence was found in Myotis lucifugus. Next, putative Helitrons were compared to all known TEs in RepBase. The putative Helitrons identified by HelitronScanner lacked homology to other known Helitrons. Finally, ORFs were identified with ORF Finder. The largest ORF from each putative Helitron was used as a BLASTp query. None of these searches identified domains associated with Helitrons (ex. Zinc-finger domains, replicase, helicase, etc. [28]) and a majority failed to recover significant hits to any known protein. Based on these results, the sequences recovered by HelitronScanner are likely artifacts of the search methodology and not true Helitrons, since these loci are single-copy, present in the Myotis lucifugus genome, lack homology to other known Helitrons, and lack ORFs expected in Helitrons.

To identify lineage-specific elements, the validated TEs were compared to all known genomes in the NCBI genomes database and classified as lineage-specific, ancestral, or disjunct based on the 50 best BLASTn hits. In all, six elements were specific to $M$. natalensis, five solo LTRs and one non-autonomous piggyBac. Seventeen of the validated elements were found in other vespertilionid bats, including eight transposons in the TcMariner superfamily. The best BLASTn hits for seven elements were to non-chiropteran taxa. Of these, six were cut and paste transposons (5 Tiggers and 1 piggyBac) and one was a LTR. All five Tiggers are elements previously identified in other non-chiropteran taxa and thus represent ancient transposons. One element, Tigger1_MNa shared similarity to more than twenty insertions in the brown kiwi (Apteryx australis) genome. All hits were $\geq 97 \%$ similar across $\geq 92 \%$ of the entire $M$. natalensis Tigger1_Mna element. Since our de novo analysis only masked chiropteran-specific elements, these elements, known from other non-chiropteran taxa, were not identified in the initial masking procedures. The closest BLASTn hit to the remaining nine elements was to Pteropus alecto, a pteropodid bat. The pteropodid bats are only distantly related to Miniopterus among bats and some elements likely represent subfamilies diverged from TEs in the ancestral bat genome. These elements were reclassified as "ancestral".

Individual TE insertions in the $M$. natalensis genome were annotated using the final validated TE library that was combined with all known mammalian repeats in RepBase. For comparison, Myotis lucifugus, E. fuscus, and P. parnellii were processed alongside $M$. natalensis. All four bat genomes contained similar quantities of TEs ranging from 24-27.5 \% (Table 1). Class II content was more variable between species than any of the retrotransposon categories. Cut and paste transposons comprised only $1.52 \%$ of the $M$. natalensis genome and less than $0.01 \%$ was derived from Helitrons. In general, DNA transposon content in M. natalensis was more similar to the outgroup, P. parnellii, than to the vespertilionids (Table 1). The repeat accumulation profile for $M$. natalensis (Fig. 1a) indicates that a significant majority of Class II elements are heavily mutated when compared to the presumed ancestral sequence, indicating long periods of inactivity within the genome. In fact, TEs in the M. natalensis genome appear to be accumulating less rapidly than in the past. $M$. natalensis and P. parnellii (Fig. 1b) both show declining accumulation of Class II elements and negligible Helitron content. Both vespertilionid bats show appreciable levels of Helitron content and recent accumulation of cut and paste elements (Fig. 1c and d).

In addition to accumulation profiles, average genetic distances between consensus elements and TE loci were calculated for all DNA transposons that occupied more than $10 \mathrm{~Kb}$ in any of the bat genomes examined (Additional file 1: Table S1). In all, 248 different DNA transposons met these criteria. A portion of this data is presented in Table 2. Genetic distances can be used as a relative metric for age and combined with presence or absence in other species to understand TE dynamics within this group. The most common superfamily of TEs in the genomes examined were hATs (133 of 248 elements). Generally, hATs fell into two categories; 1 ) they were present in some combination of vespertilionid genomes or, 2) they were found in M. natalensis and also identified in the vesper bats and the outgroup, $P$. parnellii. Based on genetic distance, nhAT-100_EF was the most recent hAT transposon in $M$. natalensis (Table 2). This transposons, nhAT-100_EF, was present in all four taxa examined and genetic distances fell within a limited range (18.69-19.75\%). Helitrons followed a similar pattern to hATs; the two Helitrons identified in $M$. natalensis were shared by all three other species and heavily mutated (Table 2). TcMariner transposons, in general, were shared among all analyzed taxa, with a limited number of exceptions. Two elements were not identified in P. parnellii. Four older elements with average genetic distances greater than $23 \%$ were found only in P. parnellii. Interestingly this analysis, which relied on RepeatMasker searches, identified a single element restricted to $M$. natalensis (nTIGGER-7_MNa). The BLAST searches used to identify lineage-specific repeats (described above) identified a single homologous sequence in another bat, Rhinolophus ferrumequinum (99\% query coverage, $85 \%$ identity, 2e-57 E value), but not to other vesper bats. It is possible that this element is specific to $M$. natalensis since it was only found at one locus in one other species. In either case, since $R$. ferrumeguinum was not in our RepeatMasker searches, the distribution of this element among the taxa examined appears reasonable. Finally, most unclassified 
Table 1 Transposable element content. The number of bases and percent of the genome derived from transposable elements was calculated in four species of bats. The percentage of the genome occupied by transposable elements was calculated based on the total genome size, excluding ambiguous regions or scaffold gaps ("N"s)

\begin{tabular}{|c|c|c|c|c|c|c|c|c|}
\hline \multirow[t]{2}{*}{ Classification } & \multicolumn{2}{|c|}{ Miniopterus natalensis } & \multicolumn{2}{|c|}{ Myotis lucifugus } & \multicolumn{2}{|l|}{ E. fuscus } & \multicolumn{2}{|l|}{ P. parnelli } \\
\hline & Bases & Percentage & Bases & Percentage & Bases & Percentage & Bases & Percentage \\
\hline Transposable elements & $415,627,321$ & $23.95 \%$ & $518,680,444$ & $27.50 \%$ & $478,933,702$ & $26.58 \%$ & $383,285,246$ & $24.76 \%$ \\
\hline Class I Retrotransposons & $388,593,157$ & $22.39 \%$ & $424,243,455$ & $22.50 \%$ & $383,040,593$ & $21.26 \%$ & $346,459,100$ & $22.37 \%$ \\
\hline Long Terminal Repeats & $69,316,646$ & $4.00 \%$ & $72,931,404$ & $3.88 \%$ & $71,532,426$ & $3.97 \%$ & $68,573,030$ & $4.43 \%$ \\
\hline ERV & $1,092,720$ & $0.06 \%$ & $1,038,965$ & $0.06 \%$ & $1,149,410$ & $0.06 \%$ & $1,499,592$ & $0.10 \%$ \\
\hline ERV1 & $23,526,841$ & $1.36 \%$ & $28,053,951$ & $1.49 \%$ & $26,324,280$ & $1.46 \%$ & $19,669,702$ & $1.27 \%$ \\
\hline ERV2 & 431,937 & $0.02 \%$ & $7,857,605$ & $0.42 \%$ & $4,951,316$ & $0.27 \%$ & 391,300 & $0.03 \%$ \\
\hline ERV3 & $41,929,575$ & $2.42 \%$ & $34,495,447$ & $1.83 \%$ & $37,663,204$ & $2.09 \%$ & $45,513,437$ & $2.94 \%$ \\
\hline Gypsy & 357,161 & $0.02 \%$ & 220,101 & $0.01 \%$ & 272,666 & $0.02 \%$ & 600,987 & $0.04 \%$ \\
\hline LTR & $1,978,412$ & $0.12 \%$ & $1,265,335$ & $0.07 \%$ & $1,171,550$ & $0.07 \%$ & 898,012 & $0.05 \%$ \\
\hline Long INterspersed Elements & $241,612,217$ & $13.92 \%$ & $242,431,627$ & $12.85 \%$ & $210,106,281$ & $11.66 \%$ & $225,554,475$ & $14.57 \%$ \\
\hline L1 & $240,801,801$ & $13.88 \%$ & $241,785,916$ & $12.82 \%$ & $209,396,239$ & $11.63 \%$ & $224,541,665$ & $14.51 \%$ \\
\hline L2 & 63,018 & $0.00 \%$ & 42,706 & $0.00 \%$ & 53,584 & $0.00 \%$ & 158,866 & $0.01 \%$ \\
\hline Penelope & 3,390 & $0.00 \%$ & 2,619 & $0.00 \%$ & 1,994 & $0.00 \%$ & 3,371 & $0.00 \%$ \\
\hline R4 & 24,859 & $0.00 \%$ & 14,178 & $0.00 \%$ & 21,390 & $0.00 \%$ & 35,098 & $0.00 \%$ \\
\hline RTE & 467,222 & $0.03 \%$ & 425,242 & $0.02 \%$ & 428,374 & $0.02 \%$ & 431,357 & $0.03 \%$ \\
\hline RTEX & 246,560 & $0.01 \%$ & 156,754 & $0.01 \%$ & 198,740 & $0.01 \%$ & 376,073 & $0.02 \%$ \\
\hline $\mathrm{Tx} 1$ & 5,367 & $0.00 \%$ & 4,212 & $0.00 \%$ & 5,960 & $0.00 \%$ & 8,045 & $0.00 \%$ \\
\hline Short INterspersed Elements & $77,664,294$ & $4.47 \%$ & $108,880,424$ & $5.77 \%$ & $101,401,886$ & $5.63 \%$ & $52,331,595$ & $3.37 \%$ \\
\hline Unclassified & 141,822 & $0.01 \%$ & 113,971 & $0.01 \%$ & 119,602 & $0.01 \%$ & 197,376 & $0.01 \%$ \\
\hline tRNA & $77,501,269$ & $4.46 \%$ & $108,745,685$ & $5.76 \%$ & $101,255,190$ & $5.62 \%$ & $52,075,143$ & $3.36 \%$ \\
\hline $7 S L$ & 1,048 & $0.00 \%$ & 1,775 & $0.00 \%$ & 1,914 & $0.00 \%$ & 3,786 & $0.00 \%$ \\
\hline $5 S$ & 20,155 & $0.00 \%$ & 18,993 & $0.00 \%$ & 25,180 & $0.00 \%$ & 55,290 & $0.00 \%$ \\
\hline Class II DNA transposons & $26,535,664$ & $1.53 \%$ & $91,629,080$ & $4.85 \%$ & $92,568,583$ & $5.14 \%$ & $36,073,984$ & $2.34 \%$ \\
\hline Cut and Paste & $26,433,314$ & $1.52 \%$ & $47,434,627$ & $2.51 \%$ & $35,693,046$ & $1.98 \%$ & $35,940,177$ & $2.33 \%$ \\
\hline Kolobok & 10,135 & $0.00 \%$ & 8,065 & $0.00 \%$ & 10,145 & $0.00 \%$ & 16,113 & $0.00 \%$ \\
\hline MuDR & 13,048 & $0.00 \%$ & 12,651 & $0.00 \%$ & 13,221 & $0.00 \%$ & 21,955 & $0.00 \%$ \\
\hline PiggyBac & 366,671 & $0.02 \%$ & 261,766 & $0.01 \%$ & 941,162 & $0.05 \%$ & 117,137 & $0.01 \%$ \\
\hline TcMar-Mariner & $7,537,182$ & $0.43 \%$ & $7,941,486$ & $0.42 \%$ & $10,197,575$ & $0.57 \%$ & $11,885,374$ & $0.77 \%$ \\
\hline hAT & $18,506,278$ & $1.07 \%$ & $39,210,659$ & $2.08 \%$ & $24,530,943$ & $1.36 \%$ & $23,899,598$ & $1.55 \%$ \\
\hline Rolling circle & 102,350 & $0.01 \%$ & $44,194,453$ & $2.34 \%$ & $56,875,537$ & $3.16 \%$ & 133,807 & $0.01 \%$ \\
\hline Helitrons & 102,350 & $0.01 \%$ & $44,194,453$ & $2.34 \%$ & $56,875,537$ & $3.16 \%$ & 133,807 & $0.01 \%$ \\
\hline Unknown & 498,500 & $0.03 \%$ & $2,807,909$ & $0.15 \%$ & $3,324,526$ & $0.18 \%$ & 752,162 & $0.05 \%$ \\
\hline
\end{tabular}



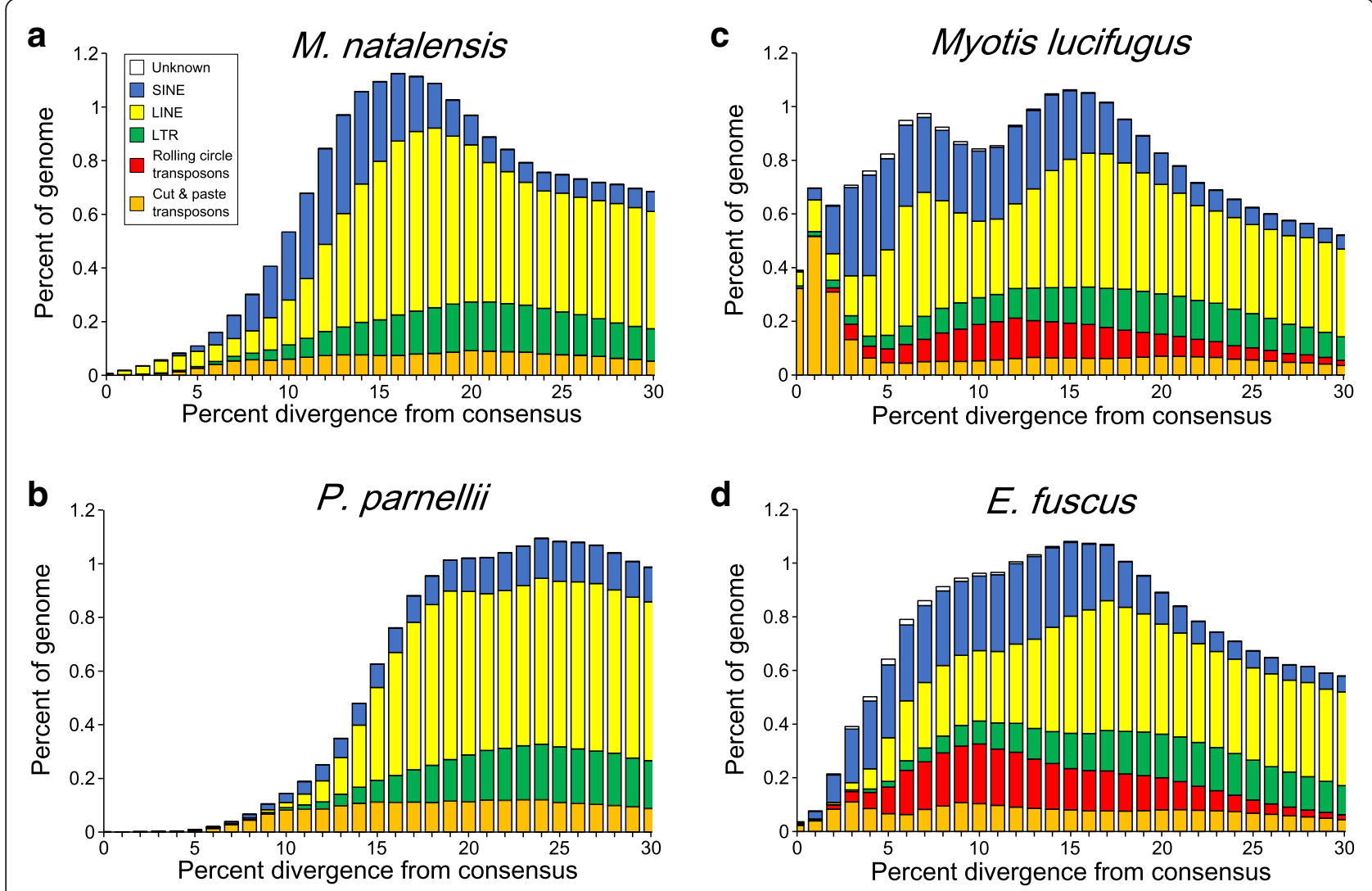

Fig. 1 Transposable element accumulation profiles in a Miniopterus natalensis, b Pteronotus parnellii, c Myotis lucifugus, and d Eptesicus fuscus. Kimura 2-parameter genetic distances were calculated between each repeat in the genome and the putative consensus for its subfamily. Distance values were binned based on transposable element type to visualize the accumulation of transposable elements over time. Due to their high mutation rate, $\mathrm{CpG}$ sites were excluded from genetic distance calculations

DNA transposons, Kolobok, and MuDRs were ancestral elements with high genetic diversity and present in all four taxa.

\section{Discussion}

Active DNA transposons are rare in mammals. To date, only the vespertilionid family of bats are known to have significant levels of active Class II elements. Miniopterus is the sole genus of the recently elevated family Miniopteridae, the sister family to Vespertilionidae [9]. Previous studies indicated that Miniopterus lacks the Helitrons found in vesper bats and may harbor limited piggyBac activity $[6,8]$. Based on these results, it has been assumed that the horizontal transfer of DNA transposons occurred in an ancestral vespertilionid bat subsequent to the divergence of Miniopterus. Complete analysis of the $M$. natalensis genome generally supports previous conclusions with slight modifications, namely that limited Class II accumulation of TcMariner and piggyback elements indicate their presence in the Miniopterus + vespertilionid ancestor. It is possible that biases introduced with sequencing chemistries, genome assembly methods, and bioinformatics analyses negatively influence the recognition of repetitive sequences. Highly repetitive sequences with low nucleotide diversity represent a significant problem for genome assembly methods. In addition, the culling of very low copy number elements $(n=<10)$ from the initial de novo repeat identification with RepeatModeler could bias estimations slightly downward. While these influences are expected to be minimal, they cannot be accurately quantified and all results should be interpreted with these caveats in mind.

\section{Species distribution of TEs identified in M. natalensis}

De novo identification of TEs and manual curation identified several elements that are novel or exhibit interesting taxonomic distributions. Tigger1_MNa shared homology with twenty insertions in the brown kiwi genome and is closely associated with the consensus sequence for TIGGER1 originally identified in the human genome. These two consensus elements (Tigger1_MNa and TIGGER1) share almost $97.5 \%$ similarity despite individual insertions being heavily mutated in the respective genomes [29] (Table 2). To demonstrate horizontal transfer between $M$. 
Table 2 The average Kimura 2-parameter, genetic distance was calculated among all insertions for each element. Highly mutable CpG sites were excluded from distance calculations

\begin{tabular}{|c|c|c|c|c|c|}
\hline \multirow[b]{2}{*}{ Super Family } & \multirow[b]{2}{*}{ Element } & \multicolumn{4}{|c|}{ Within group genetic distance (average) } \\
\hline & & Miniopterus natalensis & Myotis lucifugus & Eptesicus fuscus & Pteronotus parnellii \\
\hline$\overline{\text { hAT }}$ & nhAT-100_EF & 18.69 & 18.94 & 19.16 & 19.75 \\
\hline Helitron & Helitron1Nb_Mam & 29.66 & 30.09 & 30.45 & 30.5 \\
\hline Helitron & Helitron3Na_Mam & 32.74 & 32.87 & 33.57 & 33.93 \\
\hline PiggyBac & nPiggyBac-2_MNa & 1.35 & NA & NA & NA \\
\hline PiggyBac & piggyBac2b_Mm & NA & 1.91 & NA & NA \\
\hline PiggyBac & nPiggyBac-1_MNa & 2.03 & NA & NA & NA \\
\hline PiggyBac & piggyBac1_Mm & NA & NA & 2.18 & NA \\
\hline PiggyBac & npiggyBac-2_EF & NA & NA & 2.86 & NA \\
\hline PiggyBac & npiggyBac-1_EF & NA & 4.16 & 4.77 & NA \\
\hline PiggyBac & npiggy1_Mm & NA & NA & 5.3 & NA \\
\hline PiggyBac & piggyBac_2a_Mm & NA & 6.78 & NA & NA \\
\hline PiggyBac & nPiggyBac-3_MNa & 7.58 & NA & NA & NA \\
\hline PiggyBac & piggyBac2_Mm & 8.16 & 12.35 & 38.09 & NA \\
\hline TcMariner & nTIGGER-7_MNa & 8.08 & NA & NA & NA \\
\hline TcMariner & nTIGGER-12_MNa & 8.98 & 9.54 & 9.76 & NA \\
\hline TcMariner & nTIGGER-18_MNa & 9.99 & 9.9 & 10.33 & NA \\
\hline TcMariner & TIGGER-1_Mna & 13.63 & 14.41 & 14.6 & 15.66 \\
\hline TcMariner & TIGGER1 & 14 & 14.31 & 14.84 & 15.96 \\
\hline
\end{tabular}

Distances were only calculated if the element occupied more than 10 kilobases in a genome. For species were elements were absent or occupied less than 10 kilobases of their genome, values are given as "NA"s. A limited number of transposons are shown here. A complete table displaying the average genetic distances of all elements is provided as Additional file 1: Table S1

natalensis and the brown kiwi, an element must have a disjunct phylogenetic distribution and high sequence similarity in multiple species beyond what is expected based on divergence times [30]. The BLASTn results for Tigger1_MNa seem to support a disjunct distribution, but its heavy mutation load may be within expectations based on a neutral mutation rate and the respective divergence times of these taxa [30]. Other factors giving the appearance of a disjunct species distribution, such as sequence contamination in the kiwi genome, cannot be conclusively ruled out.

BLAST searches identified several elements specific to the $M$. natalensis genome indicating their emergence sometime in the last 37.5 [31] to $43 \mathrm{my}$ [9]. Five of these are LTRs but one non-autonomous piggyBac DNA transposon (npiggyBac-3_Mna) is specific to M. natalensis based on comparisons to all currently available genomes. npiggyBac-3_Mna was present in the $M$. natalensis genome at low frequency (577 copies). In addition to npiggyBac3_Mna, previous work noted that a small region associated with Myotis lucifugus piggyBac2_ML (bp 1,536-2,340) was also present in Miniopterus sp. [6]. Analysis of the entire $M$. natalensis genome indicates that the piggyBac2_ML fragment amplified by Ray et al. [6] is present in the $M$. natalensis genome as part of the larger piggyBac2_Mm element. RepBase does not recognize piggyBac2_ML (accessed 1 April 2016). Instead, it contains piggyBac2_Mm, the Microcebus murinus counterpart to piggyBac2_ML that is presumed to have been horizontally transferred between Microcebus murinus and Myotis lucifugus [32]. To be consistent with RepBase naming conventions, we refer to piggyBac2_ML from Ray et al. [6] as piggyBac2_Mm. In all, RepeatMasker identified fewer than 80 piggyBac2_Mm loci occupying 58,499 bps in the M. natalensis genome.

These results suggest that the PCR-based analyses of Ray et al. [6] were accurate in their identification of piggyBac2_Mm distribution among chiropterans. In that work, however, piggyBac2_Mm was absent in non-Myotis vesper bats. RepeatMasker results identify piggyBac2_Mm in E. fuscus, but in a heavily mutated and truncated form (Table 2) implying that piggyBac2_Mm elements in E. fuscus are ancestral elements misidentified as piggyBac2_Mm. The presence of closely related piggyBacs in Myotis lucifugus and M. natalensis could be explained by two possible scenarios: horizontal transfer of piggyBac2_Mm between M. natalensis and a Myotis sp. or invasion of piggyBac2_ML into the Miniopterus + vespertilionid ancestral genome, and subsequent loss in the lineage leading to Eptesicus. The genus Myotis 
occupies a basal clade within Vespertilionidae [33] meaning that if piggyBac2_Mm was present as a single or few copies, a single loss could explain the presence of piggyBac2_Mm in Myotis and M. natalensis, but not Eptesicus. Further supporting this scenario, piggyBac2_Mm contains more genetic diversity (8.16-12.35\%; Table 2) than other piggyBac elements that are limited to single species. It is likely that piggyBac2_Mm is an older subfamily of elements and may even be one of the first transposons to invade the bat genomes. On the other hand, horizontal transfer of piggyBac2_Mm involving Myotis lucifugus and Microcebus murinus (the mouse lemur) has been reported previously [32]. The distribution of these three genera (Microcebus, Miniopterus, and Myotis) all include portions of Africa and/or Madagascar, which allows for the possibility of such transfers in ancestral species (assuming similar ancestral distributions). Based on the current data, piggyBac2_Mm likely represents an invasion in an ancestral bat genome followed by a loss in E. fuscus (Fig. 2). In either case, M. natalensis, Myotis lucifugus, and E. fuscus each have lineagespecific, highly similar piggyBac transposons indicating some level of transposition in these genomes (Table 2).

\section{TE invasions in Vespertilionidae and Miniopteridae}

Just under $30 \%$ of the $M$. natalensis genome is derived from TEs. Though there is evidence of lineage-specific accumulation, the $M$. natalensis genome appears to have experienced minimal DNA transposon activity when compared to vespertilionids (Fig. 1). Around 26.5 Mb (1.5\%) of the genome is derived from Class II elements compared to $\sim 5 \%$ in the vespertilionid bats (Table 1 ). The bulk of these DNA transposon in $M$. natalensis are cut and paste DNA transposons, specifically hATs which account for $70 \%$ of all transposon content. Several observations indicate the hAT elements were deposited in a distantly related ancestor of these taxa. First, analysis of transposons in primate genomes identified significant transposon activity from TcMariners, piggyBacs, and hATs during the eutherian radiation 81-150 mya, hATs being the dominant transposon [34]. Second, the most abundant transposon in M. natalensis, hATs, were highly mutated and present in all the Vespertilionidae, M. natalensis and P. parnellii; indicating ancestral accumulation (Table 2). Third, the quantity of cut and paste transposons in M. natalensis is more similar to $P$. parnellii than its more closely related vesper relatives. Fourth, the methods used herein were capable of finding lineage-specific elements yet only one new piggyBac was identified (compared to five LTRs). Based on these results, it seems clear that the bulk of cut and paste DNA transposons were deposited prior to the Chiropteran divergence meaning that at least $70 \%$ of all DNA transposon activity in M. natalensis is ancestral.

Helitrons are not as common as cut and paste transposons in the $M$. natalensis genome, occupying less than $100 \mathrm{~Kb}$. Two Helitrons (Helitron1Nb_Mam and Helitron3Na_Mam; Table 2) appear to have been active prior to the emergence of Chiroptera based on their presence in the taxa examined. HelitronScanner, failed to identify Helitron1Nb_Mam and Helitron3Na_Mam, likely due to the high mutation load they carry ( $>30 \%$ on average; Table 2 ). The failure to identify novel Helitrons through structural searches and the low copy numbers of ancestral Helitrons identified via homology makes it reasonable to conclude
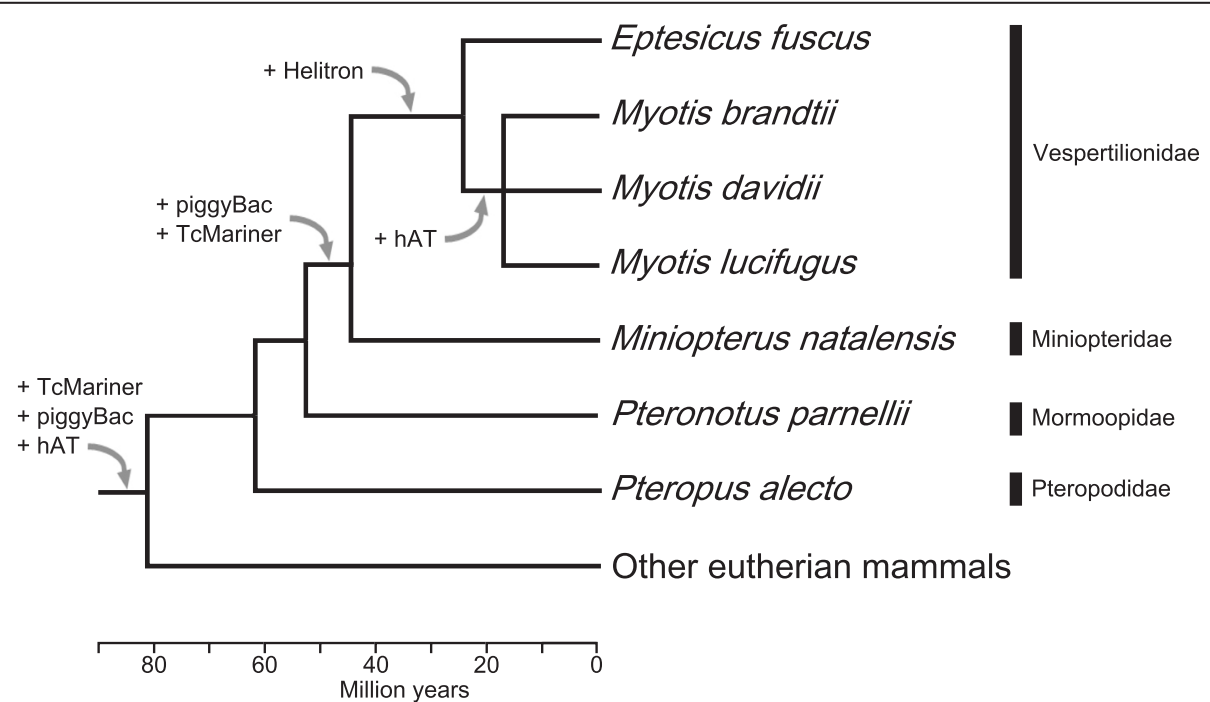

Fig. 2 A simplified tree depicting relationships among specimens examined. Time since divergence for each species is Time Tree of Life divergence estimate [39] from timetree.org. Relationships within Myotis are unresolved due to conflicting mitochondrial and nuclear phylogenies [40]. The gain of relevant, active transposons are plotted on respective nodes 
that the Helitrons invasion into the vesper bats occurred subsequent to their divergence from Miniopterus.

The lack of significant cut and paste transposon accumulation and the absence of Helitrons, allows us to place more precise taxonomic and temporal limits on the DNA transposon invasion of an ancestral bat genome (Fig. 2). The presence of a limited number of TcMariner and piggyBac families present in $M$. natalensis and the vespertilionids seems to indicate that the acquisition of DNA transposons began just before the divergence of Miniopterus and the vespertilionids. Helitrons and hATs were introduced into an ancestral vespertilionid subsequent to the divergence of Miniopterus. Lineage-specific cut and paste DNA transposons reached much higher copy numbers in the vespertilionids genomes (Fig. 1c and d) than in the M. natalensis genome (Fig. 1a).

\section{Conclusions}

The results presented here confirm and expand upon previous findings regarding the distribution of DNA transposons in bats [3-6] and suggest several avenues of research. For example, if an ancestral Miniopterus + vespertilionid bat was exposed to DNA transposons, what factors were responsible for the differential accumulation in the daughter lineages? How have genomic defense mechanisms against TEs evolved in presence/absence of DNA transposons [35]? What vectors are responsible for transferring Class II elements to these bats [36]? Finally, what role do TEs play in the generation of taxonomic and genomic diversity? The rapid diversification of the vespertilionid bats is temporally associated with the acquisition of DNA transposons [7]. Individual TE insertions are generally neutral or deleterious, but instances of exaptation are known (reviewed in [37]). Beyond individual TE insertions, TE activity in general may be beneficial, allowing species to rapidly adapt to changing environments more quickly than relying on point mutations alone [38]. Miniopterus and the vespertilionids may represent extremes in the possible diversity of mammalian TE repertoires in sister taxa. By taking advantage of these contrasting compositions, it may be possible to answer specific questions regarding TEs and their role in genome evolution.

\section{Additional files}

Additional file 1: The average Kimura 2-parameter, genetic distance was calculated among all insertions for each element. Highly mutable CpG sites were excluded from distance calculations. Distances were only calculated if the element was occupied more than 10 kilobases in a species. For species where elements were absent or occupied less than 10 kilobases of their genome, values are given as "NA"s. Calculations for all meeting these critera are shown here. Table 2 in the manuscript is reduced fascimilie of this table containing only elements specifically discussed in the text. (XLSX $22 \mathrm{~kb}$ )
Additional file 2: Novel Miniopterus natalensis transpsoable elements were identified using de novo methods and manual curation. Sequences are in FastA format. (FAS $39 \mathrm{~kb}$ )

\section{Abbreviations}

TEs, LINE, SINE, LTR, mya, ORFs, Kb

Funding

This work was supported by the National Science Foundation (DEB-1355176) to DAR. Additional support was provided by College of Arts and Sciences at Texas Tech University.

\section{Availability of data and materials}

All novel repeats discovered in the course of this study have been archived in the RepBase repeat repository. In addition, a Additional file 2 is provided with all Miniopterus natalensis repeats in FastA format.

\section{Authors' contributions}

DAR, SM, and RNP participated in all aspects of this manuscript. All authors read and approved the final manuscript.

\section{Competing interests}

The authors declare that they have no competing interests.

Received: 27 April 2016 Accepted: 13 July 2016

Published online: 22 July 2016

\section{References}

1. de Koning APJ, Gu W, Castoe TA, Batzer MA, Pollock DD. Repetitive elements may comprise over two-thirds of the human genome. PLoS Genet. 2011;7(12):e1002384. doi:10.1371/journal.pgen.1002384.

2. Lander, et al. Initial sequencing and analysis of the human genome. Nature. 2001;409(6822):860-921. http://www.nature.com/nature/journal/v409/n6822/ full/409860a0.html.

3. Ray DA, Pagan HJT, Thompson ML, Stevens RD. Bats with hATs: Evidence for recent DNA transposon activity in genus Myotis. Mol Biol Evol. 2007;24(3): 632-9. doi:10.1093/molbev/msl192

4. Pritham EJ, Feschotte C. Massive amplification of rolling-circle transposons in the lineage of the bat Myotis lucifugus. Proc Natl Acad Sci. 2007;104(6): 1895-900. doi:10.1073/pnas.0609601104.

5. Thomas J, Phillips CD, Baker RJ, Pritham EJ. Rolling-circle transposons catalyze genomic innovation in a mammalian lineage. Genome Biol Evol. 2014;6(10):2595-610. doi:10.1093/gbe/evu204.

6. Ray DA, Feschotte C, Pagan HJT, Smith JD, Pritham EJ, Arensburger P, et al. Multiple waves of recent DNA transposon activity in the bat, Myotis lucifugus. Genome Res. 2008;18(5):717-28. doi:10.1101/gr.071886.107.

7. Platt RN, Vandewege MW, Kern C, Schmidt CJ, Hoffmann FG, Ray DA. Large numbers of novel miRNAs originate from DNA transposons and are coincident with a large species radiation in bats. Mol Biol Evol. 2014;31(6): 1536-45. doi:10.1093/molbev/msu112.

8. Thomas J, Sorourian M, Ray D, Baker RJ, Pritham EJ. The limited distribution of Helitrons to vesper bats supports horizontal transfer. Gene. 2011;474(1-2): 52-8. http://dx.doi.org/10.1016/j.gene.2010.12.007.

9. Miller-Butterworth CM, Murphy WJ, O'Brien SJ, Jacobs DS, Springer MS, Teeling EC. A family matter: Conclusive resolution of the taxonomic position of the long-fingered bats, Miniopterus. Mol Biol Evol. 2007;24(7):1553-61. doi: 10.1093/molbev/msm076.

10. Eckalbar WL, Schlebusch SA, Mason MK, Gill Z, Parker AV, Booker BM et al. Transcriptomic and epigenomic characterization of the developing bat wing. Nat Genet. 2016;advance online publication. doi:10.1038/ng.3537.

11. Platt RN, Blanco-Berdugo L, Ray DA. Accurate transposable element annotation is vital when analyzing new genome assemblies. Genome Biol Evol. 2016;8(2):403-10. doi:10.1093/gbe/evw009.

12. Smit A, Hubley R. RepeatModeler Open-1.0. 2008-2015.

13. Smit A, Hubley R, P G. RepeatMasker Open-4.0. 2013-2015.

14. Altschul SF, Madden TL, Schäffer AA, Zhang J, Zhang Z, Miller W, et al. Gapped BLAST and PSI-BLAST: a new generation of protein database search programs. Nucleic Acids Res. 1997;25(17):3389-402. doi:10.1093/nar/25.17. 3389 
15. Edgar RC. MUSCLE: multiple sequence alignment with high accuracy and high throughput. Nucleic Acids Res. 2004;32(5):1792-7. doi:10.1093/nar/gkh340.

16. Hall T. BioEdit version 7.0.0. 2004.

17. Xiong W, He L, Lai J, Dooner HK, Du C. HelitronScanner uncovers a large overlooked cache of Helitron transposons in many plant genomes. Proc Natl Acad Sci. 2014;111(28):10263-8. doi:10.1073/pnas.1410068111.

18. Rice P, Longden I, Bleasby A. EMBOSS: The European Molecular Biology Open Software Suite. Trends Genet. 2000;16(6):276-7.

19. Kent WJ. BLAT-the BLAST-like alignment tool. Genome research. 2002;12(4): 656-64. doi:10.1101/gr.229202. article published online before march 2002.

20. Bao W, Kojima KK, Kohany O. Repbase Update, a database of repetitive elements in eukaryotic genomes. Mob DNA. 2015;6(1):1-6. doi:10.1186/ s13100-015-0041-9.

21. Wheeler DL, Barrett T, Benson DA, Bryant SH, Canese K, Chetvernin V, et al. Database resources of the National Center for Biotechnology Information. Nucleic Acids Res. 2007;35 suppl 1:D5-12. doi:10.1093/nar/gkl1031.

22. Wicker T, Sabot F, Hua-Van A, Bennetzen JL, Capy P, Chalhoub B, et al. A unified classification system for eukaryotic transposable elements. Nat Rev Genet. 2007:8(12):973-82

23. Kimura M. A simple method for estimating evolutionary rates of base substitutions through comparative studies of nucleotide sequences. J Mol Evol.16(2):111-20. doi:10.1007/bf01731581.

24. Xing J, Hedges DJ, Han K, Wang H, Cordaux R, Batzer MA. Alu Element Mutation Spectra: Molecular Clocks and the Effect of DNA Methylation. J Mol Biol. 2004;344(3):675-82. http://dx.doi.org/10.1016/j.jmb.2004.09.058

25. Eick GN, Jacobs DS, Matthee CA. A nuclear DNA phylogenetic perspective on the evolution of echolocation and historical biogeography of extant bats (Chiroptera). Mol Biol Evol. 2005;22(9):1869-86. doi:10.1093/molbev/msi180.

26. Ray DA, Pagan HJ, Platt RN, Kroll AR, Schaack S, Stevens RD. Differential SINE evolution in vesper and non-vesper bats. Mob DNA. 2015;6(1):1-10. doi:10. 1186/s13100-015-0038-4.

27. Platt RN, Zhang Y, Witherspoon DJ, Xing J, Suh A, Keith MS, et al. Targeted capture of phylogenetically informative Ves SINE Insertions in genus Myotis. Genome Biol Evol. 2015;7(6):1664-75. doi:10.1093/gbe/evv099.

28. Thomas J, Pritham EJ. Helitrons, the eukaryotic rolling-circle transposable elements. Microbiol Spectr. 2015;3(4). doi:10.1128/microbiolspec.MDNA30049-2014.

29. Smit AF, Riggs AD. Tiggers and DNA transposon fossils in the human genome. Proc Natl Acad Sci U S A. 1996;93(4):1443-8.

30. Schaack S, Gilbert C, Feschotte C. Promiscuous DNA: horizontal transfer of transposable elements and why it matters for eukaryotic evolution. Trends Ecol Evol. 2010;25(9):537-46. doi:10.1016/j.tree.2010.06.001.

31. Lack JB, Roehrs ZP, Stanley CE, Ruedi M, Van Den Bussche RA. Molecular phylogenetics of Myotis indicate familial-level divergence for the genus Cistugo (Chiroptera). J Mammal. 2010;91(4):976-92. doi:10.1644/09-mamm-a-192.1.

32. Pagan HJT, Smith JD, Hubley RM, Ray DA. PiggyBac-ing on a Primate genome: Novel elements, recent activity and horizontal transfer. Genome Biol Evol. 2010;2:293-303. doi:10.1093/gbe/evq021.

33. Roehrs ZP, Lack JB, Van Den Bussche RA. Tribal phylogenetic relationships within Vespertilioninae (Chiroptera: Vespertilionidae) based on mitochondrial and nuclear sequence data. J Mammal. 2010;91(5):1073-92. doi:10.1644/09-mamm-a-325.1.

34. Pace JK, Feschotte C. The evolutionary history of human DNA transposons: Evidence for intense activity in the primate lineage. Genome Res. 2007;17(4): 422-32. doi:10.1101/gr.5826307.

35. Vandewege MW, Platt RN, Ray DA, Hoffmann FG. Transposable element targeting by piRNAs in Laurasiatherians with distinct transposable element histories. Genome Biol Evol. 2016. doi:10.1093/gbe/evw078.

36. Gilbert C, Chateigner A, Ernenwein L, Barbe V, Bézier A, Herniou EA et al. Population genomics supports baculoviruses as vectors of horizontal transfer of insect transposons. Nat Commun. 2014;5. doi:10.1038/ ncomms4348.

37. Feschotte C, Pritham EJ. DNA Transposons and the Evolution of Eukaryotic Genomes. Annu Rev Genet. 2007:41:331-68. doi:10.1146/annurev.genet.40. 110405.090448.

38. Oliver KR, Greene WK. Mobile DNA and the TE-Thrust hypothesis: supporting evidence from the primates. Mob DNA. 2011;2(1):1-17. doi:10.1186/1759-8753-2-8.
39. Hedges SB, Marin J, Suleski M, Paymer M, Kumar S. Tree of Life Reveals Clock-Like Speciation and Diversification. Mol Biol Evol. 2015;32(4):835-45. doi:10.1093/molbev/msv037.

40. Stadelmann B, Lin LK, Kunz TH, Ruedi M. Molecular phylogeny of New World Myotis (Chiroptera, Vespertilionidae) inferred from mitochondrial and nuclear DNA genes. Mol Phylogenet Evol. 2007;43(1):32-48. http://dx.doi.org/10.1016/j.ympev.2006.06.019.

\section{Submit your next manuscript to BioMed Central and we will help you at every step:}

- We accept pre-submission inquiries

- Our selector tool helps you to find the most relevant journal

- We provide round the clock customer support

- Convenient online submission

- Thorough peer review

- Inclusion in PubMed and all major indexing services

- Maximum visibility for your research

Submit your manuscript at www.biomedcentral.com/submit 